\title{
Synthesis of 3,5-Diarylisoxazole Derivatives and Evaluation of in vitro Trypanocidal Activity
}

\author{
Aline A. N. de Souza, ${ }^{a}$ Viviane F. Xavier, ${ }^{a, b}$ Gleicekelly S. Coelho, ${ }^{a}$ \\ Policarpo A. Sales Junior, ${ }^{b}$ Alvaro J. Romanha, ${ }^{b}$ Silvane M. F. Murta, ${ }^{b}$ \\ Claudia M. Carneiro ${ }^{c}$ and Jason G. Taylor*,a
}

${ }^{a}$ Departamento de Química, Instituto de Ciências Exatas e Biológicas (ICEB), Universidade Federal de Ouro Preto, Campus Universitário Morro do Cruzeiro, 35400-000 Ouro Preto-MG, Brazil

${ }^{b}$ Centro de Pesquisas René Rachou, FIOCRUZ, 30190-002 Belo Horizonte-MG, Brazil

${ }^{c}$ Laboratório de Imunopatologia, Núcleo de Pesquisas em Ciências Biológicas (NUPEB), Universidade Federal de Ouro Preto, Campus Universitário Morro do Cruzeiro, 35400-000 Ouro Preto-MG, Brazil

\begin{abstract}
Chagas disease is included in the neglected tropical diseases list and is endemic to 21 Latin American countries. The two drugs currently available for treating Chagas disease are nifurtimox and benznidazole and both result in many significant side effects. The study describes the synthesis and biological evaluation of 3,5-disubstituted isoxazoles. Isoxazoles were obtained by reaction of flavones and hydroxylamine and either alkylated at the free hydroxyl group and/or nitrated at the isoxazole ring. These compounds were evaluated for their in vitro anti-Trypanosoma cruzi activity against trypomastigote and amastigote forms of the parasite in T. cruzi-infected cell lineages. Benznidazole was used as a reference compound for the in vitro assay and mammalian L929 cells were employed to evaluate cytotoxicity. A majority of the compounds tested were very active and the most active isoxazole against amastigote and trypomastigotes of $T$. cruzi was slightly more potent than the current medicine benznidazole.
\end{abstract}

Keywords: isoxazole, azole, amastigote, trypomastigote, in vitro, Chagas disease

\section{Introduction}

Discovered by Brazilian scientist Carlos Chagas in $1909,{ }^{1}$ Chagas disease has become endemic to 21 Latin American countries. ${ }^{2}$ The transmission of this disease in humans usually occurs by deposition of faeces contaminated with the protozoan hemoflagellate Trypanosoma cruzi after being bitten by the blood-sucking triatomine bugs. Almost half of those infected either have or will develop cardiomyopathy, digestive megasyndromes, or both. ${ }^{3}$ This "neglected disease" is currently treated in Brazil with benznidazole, but unfortunately, this drug causes many significant side effects. ${ }^{4}$ One of the major problems related to infectious diseases that humanity faces is the continuous mutation that microorganisms suffer and the resistance they develop to medicines. Certain strains of $T$. cruzi have

\footnotetext{
*e-mail: jason@iceb.ufop.br
}

demonstrated resistance to available drugs. ${ }^{5}$ Different approaches have been taken towards the in vitro evaluation of novel synthetic molecules that were tested for anti T. cruzi activity. The epimastigote form that is present in the midgut vector has been used for assessing anti-parasitic activity. ${ }^{6-8}$ The fact that the intracellular amastigotes forms of $T$. cruzi are present in the vertebrate host during the acute and chronic phases of the disease makes the use of this form of the parasite very attractive as screening method for the evaluation of anti $T$. cruzi activity. ${ }^{9-11}$ The trypomastigote form enters through the bite wound and is therefore initially present in the blood and, for this reason, the trypomastigote form has also been utilized for in vitro screening. ${ }^{12-14}$ Following the guidelines proposed by the Fiocruz Program for Research and Technological Development on Chagas Disease Initiative, the whole cell-based screening methodology was utilized in the present study. ${ }^{15,16}$ This allows us to study the parasite forms that are responsible for 
human infection, analyse novel compounds for anti T. cruzi activity in infected cells whilst at the same time monitoring their effects on amastigotes and trypomastigotes in the same system. Different azole derivatives such as imidazoles, ${ }^{9,17}$ thiazoles, ${ }^{12,18}$ 1,3,4-oxadiazoles, ${ }^{19}$ 1,2,4-oxadiazoles ${ }^{20}$ and 1,2,3-triazoles ${ }^{21}$ have been evaluated for their anti T. cruzi activity (Figure 1).

Isoxazoles and their derivatives are considered important heterocyclic compounds due to their prolific biological properties. ${ }^{22}$ This heterocycle can be encountered in the chemical structure of approved medicines such as Leflunomide, Cloxaciline, Sulfisoxazol, Isocarboxazide, Broxaterole and Sitaxentane amongst others. To the best of our knowledge, isoxazoles have not been explored for their anti T. cruzi activity. Motivated by the need to develop more efficient drug candidates for Chagas disease with a less severe side effects profile, we have prepared 17 isoxazole derivatives and tested them against both amastigote and trypomastigote forms of T. cruzi in vitro.

\section{Results and Discussion}

\section{Chemistry}

3,5-Diphenylisoxazole $\mathbf{1}$, was prepared from chalcone according to literature procedures ${ }^{23}$ and used as a reference in order to determine the importance of a free hydroxyl group for anti T. cruzi activity (Scheme 1). A major challenge in the synthesis of non-symmetrical 3,5-disubstitued isoxazoles using hydroxylamine is to selectively form one isomer from a substrate that bears two electrophilic centres. The main reactions for obtaining 3,5-diarylisoxazoles involve the use of chalcones ( $\alpha-\beta$ unsaturated ketones), $\beta$-diketones, flavones and cyclocondensation of nitrile oxides with alkynes. ${ }^{24}$ The key intermediate having 3,5-diarylisoxazole framework were synthesized from flavones ${ }^{25}$ in four steps (Scheme 1). The synthesis of the target isoxazoles began with the esterification of 2-hydroxyacetophenones with substituted benzoyl chlorides to provide the corresponding esters $\mathbf{2}$ as illustrated in Scheme 1. A Baker-Venkataraman rearrangement ensued in the presence of $\mathrm{KOH}$ to afford 1,3-diketones, which when isolated by precipitation and filtration, were immediately subjected to a condensation reaction under refluxing acetic acid to provide flavones 3 . Many of the ester, 1,3-diketone and flavone intermediates are known compounds and were thus confirmed by comparison of their melting point and nuclear magnetic resonance (NMR) spectral data with literature values. All data for these intermediates were in complete accordance with literature values. Flavones $\mathbf{3}$ were reacted with hydroxylamine to afford the required isoxazoles 4 in reasonable yields albeit, on occasion, mixtures of isomers were formed. The two isomers were either purified by recrystallization, separated by column chromatography or were immediately subjected to a Williamson type $O$-alkylation reaction to provide the alkylated products 5-9 in good yield. The spectroscopic data of known 3,5-diarylisoxazoles were in accordance with those previously reported. Compounds $\mathbf{5}, \mathbf{6 a - d}, \mathbf{7 a - c}, \mathbf{8}, \mathbf{9}$, $\mathbf{1 0}$ are novel and were therefore characterized by Fourier transform infrared spectroscopy (FTIR), NMR and mass spectrometry (MS).

It has been well documented that benznidazole and nifurtimox are prodrugs that require nitroreductase<smiles>Cc1cccc2c1CCC2=NNc1nc(-c2ccc(Cl)cc2)cs1</smiles><smiles>O=C(Cn1cc(-c2ccc([N+](=O)[O-])cc2)nn1)NCc1ccccc1</smiles><smiles>CC(=O)c1ccc(C2=NN(C(C)=O)C(c3ccc([N+](=O)[O-])s3)O2)cc1</smiles><smiles>O=[N+]([O-])c1cccc2c(Cl)nnc(CCc3c[nH]cn3)c12</smiles>

$\mathrm{IC}_{50}[\mu \mathrm{m}]: 4-6$

trypomastigote/amastigote

Figure 1. Selected examples of azole compounds with anti T. cruzi activity. 
<smiles>O=C(/C=C/c1ccccc1)c1cccc(-c2cc(-c3ccccc3)no2)c1</smiles>

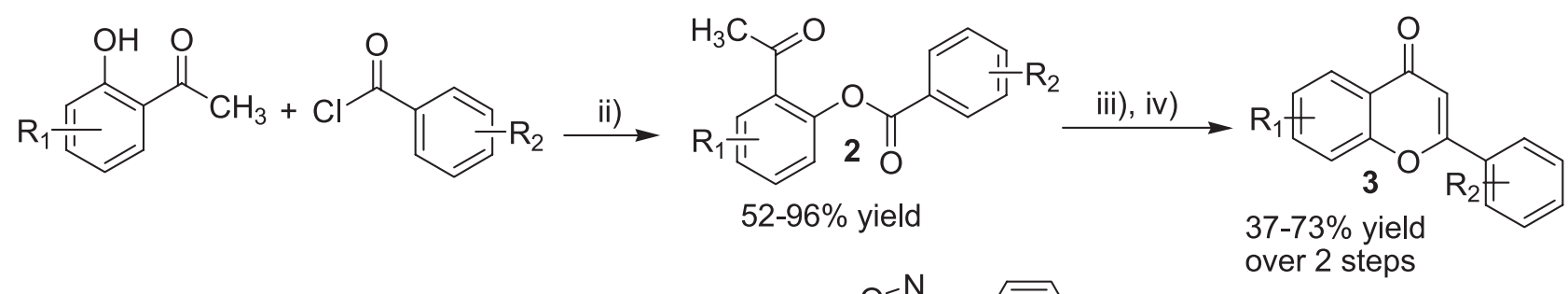

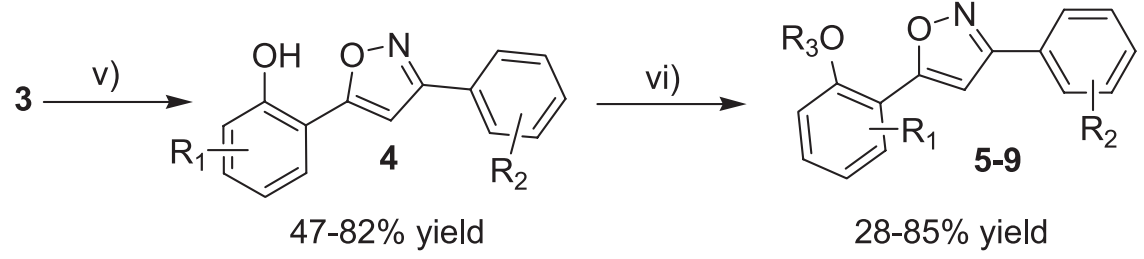

Scheme 1. Synthetic route for the preparation of isoxazole derivatives: (i) $\mathrm{NaOH}, \mathrm{NH}_{2} \mathrm{OH} . \mathrm{HCl}$, DMSO, $100{ }^{\circ} \mathrm{C}, 8 \mathrm{~h}$; (ii) pyridine, rt, $1 \mathrm{~h}$; (iii) pyridine, $\mathrm{KOH}, 50{ }^{\circ} \mathrm{C}, 1 \mathrm{~h}$; (iv) $\mathrm{AcOH}, \mathrm{H}_{2} \mathrm{SO}_{4}$, reflux, $1 \mathrm{~h}$; (v) $\mathrm{NH}_{2} \mathrm{OH}$. HCl, pyridine, reflux, $14 \mathrm{~h}$; (vi) $\mathrm{R}_{3} \mathrm{X}\left(\mathrm{X}=\mathrm{Br}\right.$ or I), acetone, $\mathrm{K}_{2} \mathrm{CO}_{3}, 60{ }^{\circ} \mathrm{C}, 18 \mathrm{~h}$.

catalyzed activation within the parasite to have trypanocidal effects. We synthesised a nitro bearing isoxazole for the purpose of evaluating possible enhancement in activity via this known mode of action involving nitroreductase. Compound 9 was nitrated in the presence of acetic anhydride and nitric acid to provide novel compound $\mathbf{1 0}$ in $32 \%$ yield (Scheme 2 ).

Biology

Once the final products were purified and fully characterized, we carried out in vitro bioassays using trypomastigote and amastigote forms of Tulahuen-strain T. cruzi, using the method described by Romanha et al. ${ }^{15}$ Benznidazole was used as positive control against $T$. cruzi and cytotoxicity was determined in mammalian L929 cells (Table 1).

Our reference compound provided an $\mathrm{IC}_{50}(50 \%$ inhibitory concentration) of $3.8 \mu \mathrm{M}$ and gave a selectivity factor (SI) of 625 and was used as a benchmark value for assessing the potency and selectivity of the isoxazole derivatives. Many of the compounds described herein were active at the tested concentrations. A comparison of 1 with $4 \mathbf{a}$ revealed that the presence of the free hydroxyl group increases potency significantly without rendering the 3,5-diarylisoxazole more cytotoxic. Introducing electron donating groups on to the unsubstituted phenyl ring of 4a did not result in any remarkable improvements in anti T. cruzi activity, but did, however, provide more cytotoxic compounds (compare $\mathbf{4 a}$ to $\mathbf{4 b}-\mathbf{c}$ ). Completely substituting the benzene ring for a furan moiety $\mathbf{4 d}$ gave similar results to $4 \mathbf{b}$ and $4 \mathbf{c}$. Next, we evaluated the effect of substituents on the phenol ring and observed almost a doubling in potency with the inclusion of two chlorine atoms (compare $\mathbf{4 b}$ to 4e). There may not necessarily be any important donor hydrogen bonding interactions associated with the free hydroxyl group of the phenol ring. Indeed, methylation of $\mathbf{4} \mathbf{c}$ afforded compound $\mathbf{5}$ and a comparison of their in vitro activities suggests that potency can be improved when a bulkier substituent in the ortho position is present. To test this hypothesis, compounds 6-8 were prepared by benzylation of their corresponding 2-(5-arylisoxazol-3-yl) phenols. In general, the presence of electron withdrawing groups such as fluorine or nitro groups on either aromatic ring gave the best in vitro activities and selectivities. In particular, compounds $6 \mathbf{b}, 7 \mathbf{b}$ and 8 were the stand out lead compounds and demonstrated very similar anti T. cruzi activity to reference compound benznidazole. Narsaiah and<smiles>CCOc1ccccc1-c1onc(-c2ccc(Cl)cc2)c1[N+](=O)[O-]</smiles> 
Table 1. In vitro trypanocidal activity, cytotoxicity and selectivity index of bioactive isoxazoles

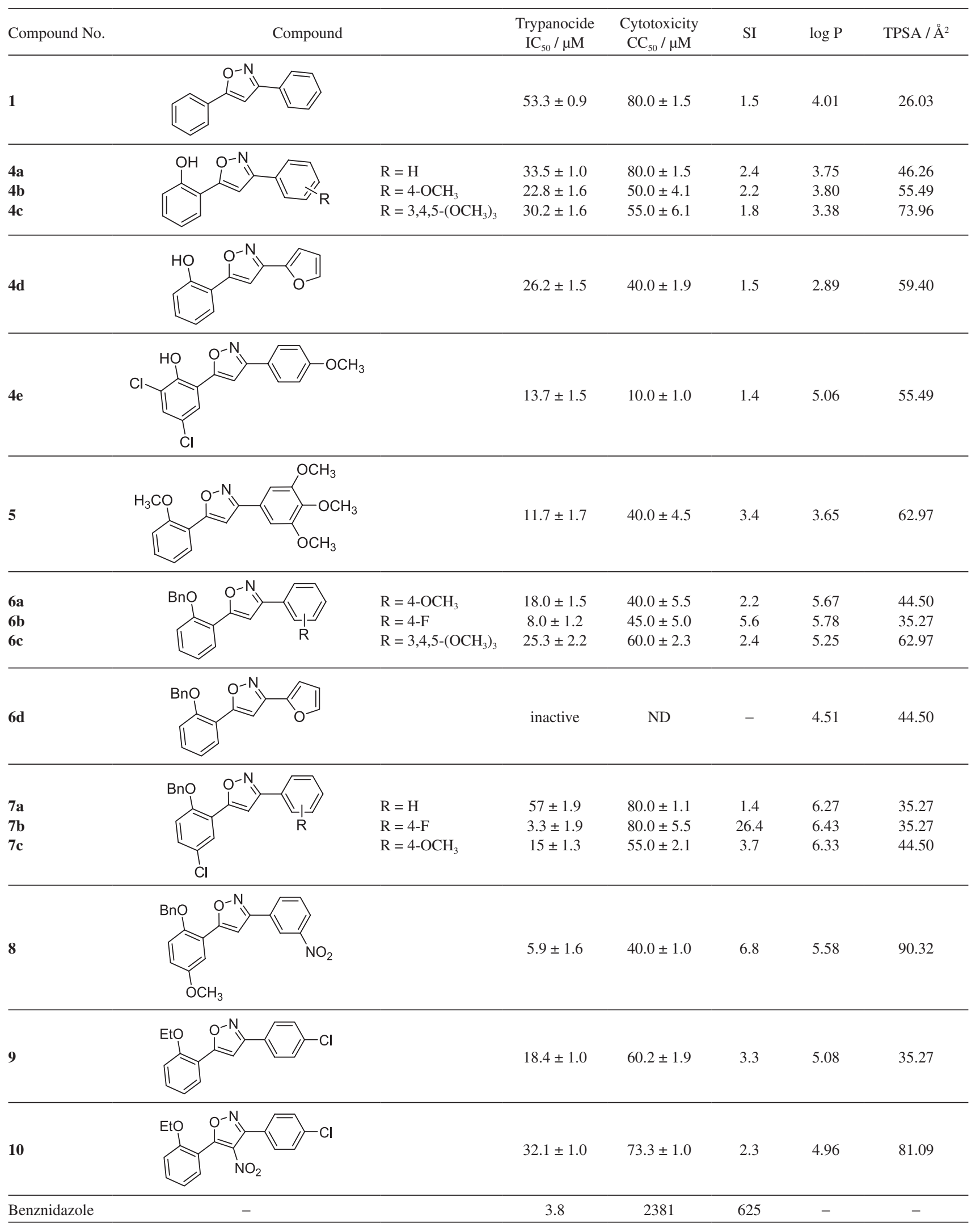

$\mathrm{IC}_{50}: 50 \%$ inhibitory concentration; $\mathrm{CC}_{50}: 50 \%$ cytotoxic concentration; ND: not determined; SI: selectivity index calculated from $\mathrm{CC}_{50} / \mathrm{IC}_{50}$; $\mathrm{TPSA}$ total polar surface area. 
co-workers ${ }^{26}$ have reported that 5-(3-alkylquinolin-2-yl)3 -aryl isoxazole derivatives screened against four human cancer cell lines (A549, COLO 205, MDA-MB 231 and PC-3) display potent cytotoxicity against all the cell lines at $\mathrm{IC}_{50}$ values of $<12 \mu \mathrm{M}$. Given the similarity in chemical structure with isoxazoles $\mathbf{4 - 9}$, the cytotoxicity profile of the compounds described in this report is supported by this literature precedence. As a consequence, the selectivity of the tested compounds was hampered by their cytotoxicity towards mammalian cells. Activation of nitroheterocyclic drugs, such as benznidazole, by T. cruzi has been associated with the formation of reactive radical species responsible for the death of the parasite. For this reason, we synthesised a nitro bearing isoxazole $\mathbf{1 0}$ for the purpose of evaluating possible enhancement in activity via this known mode of action. Surprisingly, both anti T. cruzi activity and cytotoxicity were lower than the parent compound $\mathbf{9}$ and this result suggests that nitro-isoxazoles are not activated by nitroreductase like their imidazole (benznidazole) and furan (nifurtimox) counterparts. The physicochemical drug descriptors of the molecular properties for the synthesized compounds were calculated by Molinspiration software. ${ }^{27}$ The partition coefficient (log P: octanol/water partition coefficient) describes the equilibrium distribution between two liquid phases such as octanol and water and the total polar surface area (TPSA) is a measure of the extent of the molecules exposed polar area. No linear correlations between hydrophobicity and bioactivity were observed. On the other hand, all $\log \mathrm{P}$ values for the most bioactive compounds were found to be slightly greater than $\mathbf{5}$ (Lipinski's rule of five). The largest TPSA values were all below the limit of $140 \AA^{2}$ (Lipinski's rule of five); based on the TPSA value alone, the most potent compounds, $\mathbf{6 b}$ and $\mathbf{7 b}$, would be expected to perform better in permeating the parasites cell membranes. Thus, these results suggest that penetration of cell membranes is not the critical factor determining anti T. cruzi activity.

\section{Conclusions}

In conclusion, although the potency of many of the isoxazole derivatives was less active than the positive control, anti $T$. cruzi activity was significantly improved by the inclusion of fluorine group on to either the phenol of phenyl ring of the 3,5-diarylisoxazole. In one case, the isoxazole derivative $\mathbf{7 b}$ was slightly more potent than benznidazole. Further studies and investigations by colorimetric methods and indirect analysis by light microscopy are ongoing in order to discern the mechanism of action of the most potent lead compounds uncovered in the present study.

\section{Experimental}

All commercial reagents were used as received. Anhydrous solvents were purchased from Sigma-Aldrich. Flash column chromatography was performed using silica gel 200-400 mesh. Thin layer chromatography (TLC) analysis were performed using silica gel plates, using ultraviolet (UV) light (254 nm), phosphomolybdic acid or vanillin solution for visualization. Melting points are uncorrected and were recorded on a Buchi B-540 apparatus. For NMR data, the chemical shifts are reported in $\delta(\mathrm{ppm})$ referenced to residual solvent protons and ${ }^{13} \mathrm{C}$ signals in deuterated chloroform. Coupling constants $(J)$ are expressed in hertz $(\mathrm{Hz})$. Infrared spectra were obtained on a Thermo Scientific Nicolet 380 FT-IR apparatus (600-4000 cm ${ }^{-1}$, Nicolet Instrument Corp., Madison, WI, USA) using attenuated total reflection (ATR). Mass spectra were obtained by GC-MS, Shimadzu QP-2010 Plus model (Shimadzu, Kyoto, Japan) and high resolution mass spectra (HRMS) were obtained on a Shimadzu HPLC-ESI-IT-TOF. SMILES notations of the isoxazole derivatives were inputted into the online Molinspiration software (software version v2015.01 $)^{27}$ and subjected to molecular properties prediction by Molinspiration software. Flavones were prepared according to literature methods. ${ }^{25}$

Typical experimental procedure for the synthesis of isoxazoles 4-10

In a round bottom flask $(50.0 \mathrm{~mL})$ equipped with stir bar and reflux condenser was added flavone $(2.0 \mathrm{mmol})$, pyridine $(10.0 \mathrm{~mL})$ and hydroxylamine hydrochloride $(6.0 \mathrm{mmol})$. The reaction was stirred at $100{ }^{\circ} \mathrm{C}$ for $14 \mathrm{~h}$. The reaction was then allowed to cool to room temperature and poured in an ice-water mixture $(20.0 \mathrm{~mL})$. The precipitate that formed was filtered and recrystallized from ethanol. A selection of certain isoxazoles were alkylated with either iodomethane, iodoethane or benzylbromide as follows: in a round bottom flask $(50.0 \mathrm{~mL})$ equipped with stir bar and reflux condenser were added isoxazole $(1.0 \mathrm{mmol})$, anhydrous $\mathrm{K}_{2} \mathrm{CO}_{3}(2.0 \mathrm{mmol})$ and acetone $(20.0 \mathrm{~mL})$ at $0{ }^{\circ} \mathrm{C}$. Next, the alkylhalide $(1.5 \mathrm{mmol})$ was added slowly at $0{ }^{\circ} \mathrm{C}$ with stirring and the reaction was then warmed to $60{ }^{\circ} \mathrm{C}$ and allowed to stir at this temperature overnight. Upon completion, the reaction mixture was cooled to room temperature, concentrated under reduced pressure and then taken up in to a separatory funnel containing ethyl acetate $(20.0 \mathrm{~mL})$. The organic layer was washed with distilled water $(15.0 \mathrm{~mL})$ and the organic phases were worked up in the usual way. An oily residue was obtained and purified by flash chromatography eluted 
in a gradient mixture of hexanes and ethyl acetate (1-10\% ethyl acetate in hexanes).

\section{2-(3-Phenylisoxazol-5-yl) phenol (4a)}

Product obtained as a white solid in $47 \%$ yield; $\mathrm{mp}$ 221-222 ${ }^{\circ} \mathrm{C}$ (Lit. ${ }^{12} \mathrm{mp} \mathrm{232-236}{ }^{\circ} \mathrm{C}$ ); $\mathrm{R}_{\mathrm{f}}$ (retention factor) $=0.5$ (ethyl acetate/hexane 3:7); IR (ATR) $\mathrm{v} / \mathrm{cm}^{-1} 3402$, 1614, 1456, 950, 756, 684; ${ }^{1} \mathrm{H}$ NMR (300 MHz, DMSO) $\delta$ 6.91-7.65 (m, 7H), 7.82-8.01 (m, 3H), $10.06(\mathrm{~s}, 1 \mathrm{H})$; ${ }^{13} \mathrm{C}$ NMR (75 MHz, DMSO) $\delta 101.3,114.6,115.1,117.2$, 120.2, 121.9, 127.5, 128.8, 132.1, 155.4, 161.3, 162.6, 167.0; EI (electron ionization) $\mathrm{m} / \mathrm{z}: 237$ (25\%), 207 (20\%), 117 (100\%), 73 (90\%), 63 (77\%), HRMS (ESI, electrospray ionization) $\mathrm{m} / \mathrm{z}:[\mathrm{M}+\mathrm{H}]^{+}$calcd. for $\mathrm{C}_{15} \mathrm{H}_{12} \mathrm{NO}_{2}$ : 238.0868; found: 238.0782 .

\section{2-(3-(4-Methoxyphenyl)isoxazol-5-yl)phenol (4b)}

Product obtained as a yellow solid in $78 \%$ yield. mp 208-210 ${ }^{\circ} \mathrm{C}\left(\right.$ Lit. $^{28} \mathrm{mp} 212-214{ }^{\circ} \mathrm{C}$ ); $\mathrm{R}_{\mathrm{f}}=0.4$ (ethyl acetate/ hexane 3:7); IR (ATR) v / $\mathrm{cm}^{-1} 3200,1600,1500,1450$, 1260, 1030; ${ }^{1} \mathrm{H}$ NMR (400 MHz, DMSO) $\delta 3.80$ (s, 3H), 7.00 (t, J $8.4 \mathrm{~Hz}, 1 \mathrm{H}), 7.04-7.08$ (m, 3H), 7.30 (s, 1H), 7.33 (d, J $8.4 \mathrm{~Hz}, 1 \mathrm{H}), 7.79$ (d, J 7.8 Hz, 1H), 7.90 (d, J $9.0 \mathrm{~Hz}$, $2 \mathrm{H}) ;{ }^{13} \mathrm{C}$ NMR (100 MHz, DMSO) $\delta 55.9,101.3,114.6$, $115.1,117.2,120.2,121.9,127.5,128.8,132.1,155.4$, 161.3, 162.6, 167.0; EI m/z: 267 (15\%), 252 (10\%), 236 (30\%), $108(100 \%)$; HRMS (ESI) $\mathrm{m} / z:[\mathrm{M}+\mathrm{H}]^{+}$calcd. for $\mathrm{C}_{16} \mathrm{H}_{14} \mathrm{NO}_{3}$ : 268.0974; found: 268.0967 .

\section{2-(3-(3,4,5-Trimethoxyphenyl)isoxazol-5-yl)phenol (4c)}

Product obtained as a white solid in $82 \%$ yield; $\mathrm{mp}$ 218-219 ${ }^{\circ} \mathrm{C}$ (Lit. ${ }^{29} \mathrm{mp} 235-238^{\circ} \mathrm{C}$ ); $\mathrm{R}_{\mathrm{f}}=0.3$ (ethyl acetate/ hexane 3:7); IR (ATR) v / cm-1 3160, 1600, 1500, 1420, 1250, 1010; ${ }^{1} \mathrm{H}$ NMR (400 MHz, DMSO) $\delta 3.70$ (s, 3H), 3.90 (s, 6H), 6.95 (t, J 7.5 Hz, 1H), 7.10 (d, J 8.4 Hz, 1H), 7.20 (s, 2H), 7.31-7.42 (m, 2H), $7.81(\mathrm{~d}, J 8.4 \mathrm{~Hz}, 1 \mathrm{H})$, 10.65 (brs, $1 \mathrm{H}) ;{ }^{13} \mathrm{C}$ NMR (100 MHz, DMSO) $\delta 56.8,60.8$, $101.8,104.7,114.6,117.2,120.2$, 125.0, 127.6, 132.2, 139.6, 154.0, 155.4, 163.0, 167.3; EI m/z: 327 (5\%), 312 (20\%), 296 (10\%), 170 (35\%), 93 (100\%); HRMS (ESI) $m / z:[\mathrm{M}+\mathrm{H}]^{+}$calcd. for $\mathrm{C}_{18} \mathrm{H}_{18} \mathrm{NO}_{5}: 328.1185$; found: 328.1191 .

\section{2-(3-(Furan-2-yl)isoxazol-5-yl)phenol (4d)}

Product obtained as a white solid in $55 \%$ yield; $\mathrm{mp}$ 198-199 ${ }^{\circ} \mathrm{C}$ (Lit. ${ }^{30} \mathrm{mp} 237-239{ }^{\circ} \mathrm{C}$ ); $\mathrm{R}_{\mathrm{f}}=0.5$ (ethyl acetate/ hexane 3:7); IR (ATR) v / $\mathrm{cm}^{-1} 3120,1600,1500,1370$, 1240, 960; ${ }^{1} \mathrm{H}$ NMR (500 MHz, DMSO) $\delta$ 6.71-6.72 (m, $1 \mathrm{H}), 7.0(\mathrm{t}, J 8.0 \mathrm{~Hz}, 1 \mathrm{H}), 7.10(\mathrm{~d}, J 8.0 \mathrm{~Hz}, 1 \mathrm{H}), 7.21-7.23$ $(\mathrm{m}, 2 \mathrm{H}), 7.40(\mathrm{t}, J 8.5 \mathrm{~Hz}, 1 \mathrm{H}), 7.80(\mathrm{~d}, J 8.0 \mathrm{~Hz}, 1 \mathrm{H}), 7.90$ (brs, $1 \mathrm{H}), 10.70$ (s, 1H, OH); ${ }^{13} \mathrm{C}$ NMR (125 MHz, DMSO) $\delta 100.7,111.8,112.5,114.0,117.0,119.9,127.3,132.1$, 144.2, 145.3, 155.2, 155.3, 166.8; EI m/z: 227 (55\%), 121 (100\%), 105 (70\%), 65 (90\%); HRMS (ESI) m/z: [M + H] $]^{+}$ calcd. for $\mathrm{C}_{13} \mathrm{H}_{10} \mathrm{NO}_{3}$ : 228.0661; found: 228.0656 .

2,4-Dichloro-6-(3-(4-methoxyphenyl)isoxazol-5-yl)phenol $(4 \mathrm{e})$

Product obtained as a white solid in $47 \%$ yield; $m p 206$ $208{ }^{\circ} \mathrm{C}$ (Lit. ${ }^{31} \mathrm{mp} 193{ }^{\circ} \mathrm{C}$ ); $\mathrm{R}_{\mathrm{f}}=0.4$ (ethyl acetate/hexane 3:7); IR (ATR) $v / \mathrm{cm}^{-1} 1701,1359,1224,536 ;{ }^{1} \mathrm{H}$ NMR (400 MHz, DMSO) $\delta 3.80$ (s, 3H), 7.10 (d, J 8.8 Hz, 2H), $7.40(\mathrm{~s}, 1 \mathrm{H}) ; 7.70(\mathrm{~d}, J 8.4 \mathrm{~Hz}, 1 \mathrm{H}), 7.80(\mathrm{~d}, J 8.4 \mathrm{~Hz}$, 1H), 7.90 (d, J $8.8 \mathrm{~Hz}, 2 \mathrm{H}) ;{ }^{13} \mathrm{C}$ NMR (100 MHz, DMSO) $\delta$ 55.8, 102.8, 115.0, 118.7, 121.2, 123.9, 124.4, 125.9, 128.6, 130.9, 149.8, 161.3, 162.6, 165.0; EI $\mathrm{m} / z: 335$ (10\%), 108 (100\%); HRMS (ESI) $\mathrm{m} / z:[\mathrm{M}+\mathrm{H}]^{+}$calcd. for $\mathrm{C}_{16} \mathrm{H}_{12} \mathrm{Cl}_{2} \mathrm{NO}_{3}$ : 336.0194; found: 336.0200.

\section{5-(2-Methoxyphenyl)-3-(3,4,5-trimethoxyphenyl)isoxazole} (5)

Product obtained as a white solid in $72 \%$ yield; mp $88-92{ }^{\circ} \mathrm{C} ; \mathrm{R}_{\mathrm{f}}=0.5$ (ethyl acetate/hexane 3:7); IR (ATR) $\mathrm{v} / \mathrm{cm}^{-1} 1600,1500,1460,1240,1120 ;{ }^{1} \mathrm{H} \mathrm{NMR}(500 \mathrm{MHz}$, $\left.\mathrm{CDCl}_{3}\right) \delta 3.90(\mathrm{~s}, 3 \mathrm{H}), 4.00(\mathrm{~s}, 6 \mathrm{H}), 4.05(\mathrm{~s}, 3 \mathrm{H}), 7.06-7.15$ $(\mathrm{m}, 5 \mathrm{H}), 7.45-7.48(\mathrm{~m}, 1 \mathrm{H}), 8.05(\mathrm{~d}, J 7.5 \mathrm{~Hz}, 1 \mathrm{H})$; ${ }^{13} \mathrm{C} \mathrm{NMR}\left(75 \mathrm{MHz}, \mathrm{CDCl}_{3}\right) \delta 55.7,56.4,61.0,101.4,104.3$, $111.3,116.5,121.0,125.0,127.8,131.3,139.6,153.6$, 156.2, 162.9, 166.5; EI m/z: 341 (100\%), 326 (50\%), 135 $(90 \%)$; HRMS (ESI) $m / z:[\mathrm{M}+\mathrm{H}]^{+}$calcd. for $\mathrm{C}_{19} \mathrm{H}_{20} \mathrm{NO}_{5}$ : 342.1341; found: 342.1333 .

5-(2-(Benzyloxy)phenyl)-3-(4-methoxyphenyl)isoxazole (6a)

Product obtained as a yellow solid in $62 \%$ yield; $\mathrm{mp}$ 95-98 ${ }^{\circ} \mathrm{C} ; \mathrm{R}_{\mathrm{f}}=0.6$ (ethyl acetate/hexane 3:7); IR (ATR) $\mathrm{v} / \mathrm{cm}^{-1}$ 2925, 1608, 1512, 1458, 1249; ${ }^{1} \mathrm{H}$ NMR (400 MHz, $\left.\mathrm{CDCl}_{3}\right) \delta 3.90(\mathrm{~s}, 3 \mathrm{H}), 5.30(\mathrm{~s}, 2 \mathrm{H}), 7.00(\mathrm{~d}, J 9.2 \mathrm{~Hz}$, $2 \mathrm{H}), 7.06(\mathrm{~s}, 1 \mathrm{H}), 7.10-7.16(\mathrm{~m}, 2 \mathrm{H}), 7.40-7.50(\mathrm{~m}$, $4 \mathrm{H}), 7.55(\mathrm{~d}, J 8.4 \mathrm{~Hz}, 2 \mathrm{H}), 7.70(\mathrm{~d}, J 8.8 \mathrm{~Hz}, 2 \mathrm{H})$, $8.10(\mathrm{~d}, J 7.6 \mathrm{~Hz}, 1 \mathrm{H}) ;{ }^{13} \mathrm{C}$ NMR $\left(100 \mathrm{MHz}, \mathrm{CDCl}_{3}\right)$ $\delta$ 55.4, 70.7, 101.6, 112.6, 114.2, 117.0, 121.2, 122.1, 127.7, 127.8, 128.1, 128.4, 128.7, 131.0, 136.4, 155.3, 160.8, 162.5, 166.0; EI $m / z$ : 357 (10\%), 91 (100\%); HRMS (ESI) $m / z$ : $[\mathrm{M}+\mathrm{H}]^{+}$calcd. for $\mathrm{C}_{23} \mathrm{H}_{20} \mathrm{NO}_{3}$ : 358.1443; found: 358.1488.

5-(2-(Benzyloxy)phenyl)-3-(4-fluorophenyl)isoxazole (6b)

Product obtained as a white solid in $27 \%$ yield; mp 145-148 ${ }^{\circ} \mathrm{C} ; \mathrm{R}_{\mathrm{f}}=0.66$ (ethyl acetate/hexane 3:7); IR (ATR) $\mathrm{v} / \mathrm{cm}^{-1}$ 1500, 1442, 1236, 754, 698; ${ }^{1} \mathrm{H}$ NMR (400 MHz, $\left.\mathrm{CDCl}_{3}\right) \delta 5.30(\mathrm{~s}, 2 \mathrm{H}), 7.05(\mathrm{~s}, 1 \mathrm{H}), 7.11-7.17(\mathrm{~m}, 4 \mathrm{H})$, 7.40-7.55 (m, 6H), 7.75-7.80 (m, 2H), 8.10 (d, J 7.6 Hz, $1 \mathrm{H}) ;{ }^{13} \mathrm{C} \mathrm{NMR}\left(100 \mathrm{MHz}, \mathrm{CDCl}_{3}\right) \delta 70.8,101.6,112.6$, 
$116.0\left(\mathrm{~d}, J_{\mathrm{C}-\mathrm{F}} 11 \mathrm{~Hz}\right), 116.7,121.3,125.7\left(\mathrm{~d}, J_{\mathrm{C}-\mathrm{F}} 3 \mathrm{~Hz}\right)$, $127.7,127.8,128.4,128.5\left(\mathrm{~d}, J_{\mathrm{C}-\mathrm{F}} 9 \mathrm{~Hz}\right), 128.8,131.2$, 136.3, 155.4, 162.1, 163.7 (d, $\left.J_{\mathrm{C}-\mathrm{F}} 248 \mathrm{~Hz}\right), 166.4$; EI $\mathrm{m} / z$ : 345 (10\%), 91 (100\%); HRMS (ESI) $\mathrm{m} / z:[\mathrm{M}+\mathrm{H}]^{+}$calcd. for $\mathrm{C}_{22} \mathrm{H}_{17} \mathrm{FNO}_{2}$ : 346.1243; found: 346.1231 .

\section{5-(2-(Benzyloxy)phenyl)-3-(3,4,5-trimethoxyphenyl) isoxazole (6c)}

Product obtained as a white solid in $78 \%$ yield; $\mathrm{mp}$ $148-153{ }^{\circ} \mathrm{C} ; \mathrm{R}_{\mathrm{f}}=0.5$ (ethyl acetate/hexane 3:7); IR (ATR) $v / \mathrm{cm}^{-1} 1600,1500,1450,1140,1000 ;{ }^{1} \mathrm{H}$ NMR $(300 \mathrm{MHz}$, $\left.\mathrm{CDCl}_{3}\right) \delta 3.86$ (s, 6H), 3.88 (s, 3H), 5.20 (s, 2H), 6.90-7.01 $(\mathrm{m}, 2 \mathrm{H}), 7.11-7.16(\mathrm{~m}, 2 \mathrm{H}), 7.37-7.46(\mathrm{~m}, 4 \mathrm{H}), 7.55(\mathrm{~d}$, $J 7.2 \mathrm{~Hz}, 2 \mathrm{H}), 8.03(\mathrm{~d}, J 7.8 \mathrm{~Hz}, 1 \mathrm{H}) ;{ }^{13} \mathrm{C}$ NMR $(75 \mathrm{MHz}$, $\left.\mathrm{CDCl}_{3}\right) \delta 56.1,60.9,70.7,101.7,103.8,112.3,116.6$, 121.2, 124.8, 127.7, 128.2, 128.3, 128.7, 131.2, 136.3, 139.4, 153.5, 155.4, 162.7, 166.2; EI m/z: 312 (100\%), 297 (50\%); HRMS (ESI) $m / z:[\mathrm{M}+\mathrm{H}]^{+}$calcd. for $\mathrm{C}_{25} \mathrm{H}_{24} \mathrm{NO}_{5}$ : 418.1654; found: 418.1649 .

\section{5-(2-(Benzyloxy)phenyl)-3-(furan-2-yl)isoxazole (6d)}

Product obtained as a white solid in $60 \%$ yield; $\mathrm{mp}$ $122-123^{\circ} \mathrm{C} ; \mathrm{R}_{\mathrm{f}}=0.34$ (ethyl acetate/hexane 3:7); IR (ATR) $v / \mathrm{cm}^{-1} 1600,1400,1400,1260,1020 ;{ }^{1} \mathrm{H}$ NMR $(400 \mathrm{MHz}$, $\left.\mathrm{CDCl}_{3}\right) \delta 5.30(\mathrm{~s}, 2 \mathrm{H}), 6.53-6.55(\mathrm{~m}, 1 \mathrm{H}), 6.80(\mathrm{~d}, J 3.2 \mathrm{~Hz}$, $1 \mathrm{H}), 7.10(\mathrm{~s}, 1 \mathrm{H})$, 7.15-7.40 (m, 2H), 7.48-7.51 (m, 4H), 7.56 (d, J $8.8 \mathrm{~Hz}, 2 \mathrm{H}), 7.57$ (brs, 1H), 8.10 (d, J $7.60 \mathrm{~Hz}$, $1 \mathrm{H}) ;{ }^{13} \mathrm{C}$ NMR $\left(100 \mathrm{MHz}, \mathrm{CDCl}_{3}\right) \delta 70.7,101.2,110.0$, 111.6, 112.6, 116.6, 121.2, 127.5, 128.0, 128.3, 128.7, 131.3, 136.3, 143.7, 144.8, 155.4, 155.4, 166.0; EI $\mathrm{m} / \mathrm{z}$ : $317(10 \%), 91(100 \%)$; HRMS (ESI) $\mathrm{m} / z:[\mathrm{M}+\mathrm{H}]^{+}$calcd. for $\mathrm{C}_{20} \mathrm{H}_{16} \mathrm{NO}_{3}$ : 318.1130; found: 318.1123 .

\section{5-(2-(Benzyloxy)-5-chlorophenyl)-3-phenylisoxazole (7a)}

Product obtained as a white solid in $79 \%$ yield; $\mathrm{mp}$ 196-196 ${ }^{\circ} \mathrm{C} ; \mathrm{R}_{\mathrm{f}}=0.34$ (ethyl acetate/hexane 3:7); IR (ATR) $v / \mathrm{cm}^{-1} 1600,1400,1360,1240,1020 ;{ }^{1} \mathrm{H}$ NMR $(300 \mathrm{MHz}$, $\left.\mathrm{CDCl}_{3}\right) \delta 5.20(\mathrm{~s}, 2 \mathrm{H}), 7.0(\mathrm{~d}, J 8.7 \mathrm{~Hz}, 1 \mathrm{H}), 7.10(\mathrm{~s}, 1 \mathrm{H})$, 7.33-7.36 (d, J 2.4, 9.0 Hz, 1H), 7.43-7.47 (m, 8H), 7.74$7.77(\mathrm{~m}, 2 \mathrm{H}), 8.0(\mathrm{~d}, J 2.40 \mathrm{~Hz}, 1 \mathrm{H}) ;{ }^{13} \mathrm{C} \mathrm{NMR}(75 \mathrm{MHz}$, $\left.\mathrm{CDCl}_{3}\right) \delta 70.1,102.5,113.8,118.1,126.4,126.7,127.4$, $127.6,128.5,128.8,128.8,129.2,129.9,130.6,135.8$, 153.8, 163.0, 164.9; EI $m / z: 361$ (5\%), 91 (100\%), HRMS (ESI) $m / z:[\mathrm{M}+\mathrm{H}]^{+}$calcd. for $\mathrm{C}_{22} \mathrm{H}_{17} \mathrm{ClNO}_{2}: 362.0948$; found: 362.0965 .

\section{5-(2-(Benzyloxy)-5-chlorophenyl)-3-(4-fluorophenyl) isoxazole $(\mathbf{7 b})$}

Product obtained as a white solid in $85 \%$ yield; $\mathrm{mp}$ $192-193{ }^{\circ} \mathrm{C} ; \mathrm{R}_{\mathrm{f}}=0.3$ (ethyl acetate/hexane 3:7); IR (ATR) $\mathrm{v} / \mathrm{cm}^{-1} 1600,1500,1440,1260,1240,1020,740 ;{ }^{1} \mathrm{H}$ NMR $\left(300 \mathrm{MHz}, \mathrm{CDCl}_{3}\right) \delta 5.20(\mathrm{~s}, 2 \mathrm{H}), 7.00(\mathrm{~s}, 2 \mathrm{H}), 7.10(\mathrm{~d}$, $J 8.7 \mathrm{~Hz}, 2 \mathrm{H}), 7.32-7.35$ (dd, $J 9.0,2.4 \mathrm{~Hz}, 1 \mathrm{H}), 7.40-$ $7.47(\mathrm{~m}, 5 \mathrm{H}), 7.68-7.72(\mathrm{~m}, 2 \mathrm{H}), 8.0(\mathrm{~d}, J 2.40 \mathrm{~Hz}, 1 \mathrm{H})$; ${ }^{13} \mathrm{C} \mathrm{NMR}\left(75 \mathrm{MHz}, \mathrm{CDCl}_{3}\right) \delta 71.1,102.3,113.9,116.1(\mathrm{~d}$, $\left.J_{\mathrm{C}-\mathrm{F}} 21.75 \mathrm{~Hz}\right), 117.8,126.4,127.4,127.6,128.5\left(\mathrm{~d}, J_{\mathrm{C}-\mathrm{F}}\right.$ $1.5 \mathrm{~Hz}), 128.6,128.8,130.8,135.8,153.8,162.7,162.1$, 165.1, 165.4; EI m/z: 379 (15\%), 120 (25\%), 91 (100\%), HRMS (ESI) $m / z:[\mathrm{M}+\mathrm{H}]^{+}$calcd. for $\mathrm{C}_{22} \mathrm{H}_{16} \mathrm{ClFO}_{2}$ : 380.0854; found: 380.0853 .

5-(2-(Benzyloxy)-5-chlorophenyl)-3-(4-methoxyphenyl) isoxazole (7c)

Product obtained as a white solid in $32 \%$ yield; $\mathrm{mp}$ 174-174 ${ }^{\circ} \mathrm{C} ; \mathrm{R}_{\mathrm{f}}=0.4$ (ethyl acetate/hexane 3:7); IR (ATR) $\mathrm{V} / \mathrm{cm}^{-1} 1600,1500,1360,1250,1020,710 ;{ }^{1} \mathrm{H}$ NMR $\left(300 \mathrm{MHz}, \mathrm{CDCl}_{3}\right) \delta 3.90(\mathrm{~s}, 3 \mathrm{H}), 5.20(\mathrm{~s}, 2 \mathrm{H}), 6.90-7.00$ (m, 4H), 7.30-7.33 (dd, J 2.4, $9.0 \mathrm{~Hz}, 1 \mathrm{H}), 7.40-7.50$ $(\mathrm{m}, 5 \mathrm{H}), 7.70(\mathrm{~d}, J 8.1 \mathrm{~Hz}, 2 \mathrm{H}), 8.00(\mathrm{~d}, 1 \mathrm{H}) ;{ }^{13} \mathrm{C} \mathrm{NMR}$ $\left(75 \mathrm{MHz}, \mathrm{CDCl}_{3}\right) \delta 55.3,71.1,102.3,113.8,114.2,118.1$, $121.7,126.4,127.4,127.7,128.1,128.5,128.8,130.5$, 135.0, 153.7, 160.9, 162.6, 164.6; EI m/z: 391 (20\%), 108 (25\%), 91 (100\%); HRMS (ESI) $m / z:[\mathrm{M}+\mathrm{H}]^{+}$calcd. for $\mathrm{C}_{23} \mathrm{H}_{19} \mathrm{ClNO}_{3}$ : 392.1053; found: 392.1044 .

5-(2-(Benzyloxy)-5-methoxyphenyl)-3-(3-nitrophenyl) isoxazole (8)

Product obtained as a white solid in $63 \%$ yield; $\mathrm{mp}$ $150-152{ }^{\circ} \mathrm{C} ; \mathrm{R}_{\mathrm{f}}=0.30$ (ethyl acetate/hexane 3:7); IR (ATR) $v / \mathrm{cm}^{-1} 1600,1500,1360,1230,1070 ;{ }^{1} \mathrm{H}$ NMR $\left(300 \mathrm{MHz}, \mathrm{CDCl}_{3}\right) \delta 3.90(\mathrm{~s}, 3 \mathrm{H}), 5.30(\mathrm{~s}, 2 \mathrm{H}), 7.0$ $(\mathrm{d}, J 9.6 \mathrm{~Hz}, 1 \mathrm{H}), 7.10(\mathrm{~s}, 1 \mathrm{H}), 7.30(\mathrm{~d}, J 9.0 \mathrm{~Hz}, 1 \mathrm{H})$, 7.33-7.43 (m, 4H), $7.50(\mathrm{~d}, J 7.8 \mathrm{~Hz}, 2 \mathrm{H}), 7.60$ (t, $J 8.1 \mathrm{~Hz}$, $1 \mathrm{H}), 8.10(\mathrm{~d}, J 8.7 \mathrm{~Hz}, 1 \mathrm{H}), 8.30(\mathrm{~d}, J 9.0 \mathrm{~Hz}, 1 \mathrm{H}), 8.70$ $(\mathrm{s}, 1 \mathrm{H}) ;{ }^{13} \mathrm{C}$ NMR $\left(75 \mathrm{MHz}, \mathrm{CDCl}_{3}\right) \delta 55.8,76.4,94.8$, 104.4, 118.6, 118.7, 119.3, 120.6, 124.5, 127.9, 128.3, 128.4, 129.7, 131.2, 134.7, 138.0, 143.4, 146.0, 148.5, 152.3, 156.7; EI $m / z: 402$ (5\%), 91 (100\%); HRMS (ESI) $m / z:[\mathrm{M}+\mathrm{H}]^{+}$calcd. for $\mathrm{C}_{23} \mathrm{H}_{19} \mathrm{~N}_{2} \mathrm{O}_{5}: 403.1294$; found: 403.1314 .

\section{5-(4-Chlorophenyl)-3-(2-ethoxyphenyl)isoxazole (9)}

Product obtained as a white solid in 57\% yield; mp $142-144{ }^{\circ} \mathrm{C} ; \mathrm{R}_{\mathrm{f}}=0.38$ (ethyl acetate/hexane 3:7); IR (ATR) $\mathrm{v} / \mathrm{cm}^{-1} 1600,1500,1460,1240,1120 ;{ }^{1} \mathrm{H}$ NMR $(300 \mathrm{MHz}$, $\left.\mathrm{CDCl}_{3}\right) \delta 1.56(\mathrm{t}, J 6.0 \mathrm{~Hz}, 3 \mathrm{H}), 4.22(\mathrm{q}, J 6.0 \mathrm{~Hz}, 2 \mathrm{H}), 7.00-$ $7.11(\mathrm{~m}, 3 \mathrm{H}), 7.38-7.47(\mathrm{~m}, 3 \mathrm{H}), 7.80(\mathrm{~d}, J 9.0 \mathrm{~Hz}, 2 \mathrm{H})$, $8.02(\mathrm{~d}, J 6.0 \mathrm{~Hz}, 1 \mathrm{H}) ;{ }^{13} \mathrm{C} \mathrm{NMR}\left(75 \mathrm{MHz}, \mathrm{CDCl}_{3}\right) \delta 14.8$, 64.1, 101.2, 111.9, 116.2, 120.8, 126.0, 127.7, 128.1, 129.1, 131.3, 135.7, 155.6, 161.9, 166.7; EI m/z: 299 (30\%), 113 (10\%), 77 (15\%); HRMS (ESI) $\mathrm{m} / \mathrm{z}:[\mathrm{M}+\mathrm{H}]^{+}$calcd. for $\mathrm{C}_{17} \mathrm{H}_{15} \mathrm{ClNO}_{2}$ : 300.0791; found: 300.0831 . 


\section{Nitration of isoxazole 9}

Synthesis of 3-(4-chlorophenyl)-5-(2-ethoxyphenyl)4-nitroisoxazole (10)

To a suspension of isoxazole $\mathbf{1 0}(230 \mathrm{mg}, 0.69 \mathrm{mmol})$ in $\mathrm{Ac}_{2} \mathrm{O}(7 \mathrm{~mL})$ was added dropwise concentrated $\mathrm{HNO}_{3}$ $(0.05 \mathrm{~mL})$ at $0{ }^{\circ} \mathrm{C}$ with stirring. A temperature range of 0 and $5{ }^{\circ} \mathrm{C}$ was maintained until addition of nitric acid was complete. Next, the reaction mixture was stirred for $70 \mathrm{~h}$ at room temperature and then poured into ice $(25 \mathrm{~mL})$ and extracted with dichloromethane $(3 \times 20 \mathrm{~mL})$. The organic extracts was dried with sodium sulfate, filtered and concentrated under vacuum. The yellow solid obtained was triturated with $\mathrm{Et}_{2} \mathrm{O}$, filtered and then purified by column chromatography (ethyl acetate/hexane 1:9) to yield $\mathbf{1 0}$ as a pale yellow solid in $32 \%$ yield; $\mathrm{mp} 193-194{ }^{\circ} \mathrm{C} ; \mathrm{R}_{\mathrm{f}}=0.36$ (ethyl acetate/hexane 1:9); IR (ATR) $v / \mathrm{cm}^{-1}$ 1504, 1429, 1346, 1286, 1089, 709; ${ }^{1} \mathrm{H}$ NMR $\left(300 \mathrm{MHz}, \mathrm{CDCl}_{3}\right) \delta 1.67$ $(\mathrm{t}, J 6.0 \mathrm{~Hz}, 3 \mathrm{H}), 4.39(\mathrm{q}, J 6.0 \mathrm{~Hz}, 2 \mathrm{H}), 7.10-7.07$ (m, 2H), 7.47 (d, J 9.0 Hz, 2H), 7.80 (d, J 9.0 Hz, 2H), 8.30 (dd, J 9.0, 3.0 Hz, 1H), 8.89 (s, 1H); ${ }^{13} \mathrm{C} \mathrm{NMR}(75 \mathrm{MHz}$, $\left.\mathrm{CDCl}_{3}\right) \delta 14.6,65.6,102.6,111.9,116.7,123.6,126.7$, 127.4, 128.0, 129.2, 136.1, 141.1, 159.7, 162.2, 164.3; EI $m / z: 344(5 \%)$, HRMS (ESI) $m / z:[\mathrm{M}+\mathrm{H}]^{+}$calcd. for $\mathrm{C}_{17} \mathrm{H}_{14} \mathrm{ClN}_{2} \mathrm{O}_{4}$ : 345.0642; found: 345.0654 .

\section{Anti-Trypanosoma cruzi activity assay}

The in vitro anti-T. cruzi activity was evaluated on L929 cells (mouse fibroblasts) infected with Tulahuen strain of the parasite expressing the Escherichia coli $\beta$-galactosidase as reporter gene according to the method described previously. ${ }^{15}$ Briefly, for the bioassay, 4,000 L929 cells were added to each well of a 96-well microtiter plate. After an overnight incubation, 40,000 trypomastigotes were added to the cells and incubated for $2 \mathrm{~h}$. Then the medium containing extracelullar parasites was replaced with $200 \mu \mathrm{L}$ of fresh medium and the plate was incubated for an additional $48 \mathrm{~h}$ to establish the infection. For $\mathrm{IC}_{50}$ determination, the cells were exposed to each synthesized compound at serial decreasing dilutions and the plate was incubated for $96 \mathrm{~h}$. After this period, $50 \mu \mathrm{L}$ of $500 \mu \mathrm{M}$ chlorophenol red beta$D$-galactopyranoside (CPRG) in $0.5 \%$ Nonidet P40 was added to each well, and the plate was incubated for 16 to $20 \mathrm{~h}$, after which the absorbance at $570 \mathrm{~nm}$ was measured. Controls with uninfected cells, untreated infected cells, infected cells treated with benznidazole at $3.8 \mu \mathrm{M}$ (positive control) or DMSO $1 \%$ were used. The results were expressed as the percentage of $T$. cruzi growth inhibition in compound-tested cells as compared to the infected cells and untreated cells. The $\mathrm{IC}_{50}$ values were calculated by linear interpolation. Quadruplicates were run in the same plate, and the experiments were repeated at least once.

\section{Supplementary Information}

Supplementary information and NMR spectra are available free of charge at http://jbcs.sbq.org.br as PDF file.

\section{Acknowledgments}

This work was supported by the Brazilian funding agency Fundação de Amparo à Pesquisa do Estado de Minas Gerais (FAPEMIG) under research grant project code APQ-01629-16. Authors gratefully acknowledge the generous financial support from the Universidade Federal de Ouro Preto (UFOP), FAPEMIG and the Conselho Nacional de Desenvolvimento Científico e Tecnológico (CNPq). The authors thank the Program for Technological Development of Tools for Health-PDTIS-FIOCRUZ for use of its facilities. The authors would also like to thank Prof Dr Robson Jose de Cassia Afonso (UFOP) and Ananda Lima Sanson (UFOP) for the excellent mass spectrometry service.

\section{References}

1. Chagas, C.; Mem. Inst. Oswaldo Cruz 1909, 1, 159.

2. Hotez, P. J.; Dumonteil, E.; Woc-Colburn, L.; Serpa, J. A.; Bezek, S.; Edwards, M. S.; Hallmark, C. J.; Musselwhite, L. W.; Flink, B. J.; Bottazzi, M. E.; PLoS Neglected Trop. Dis. 2012, 6, 1498.

3. Junior, A. R.; Rassi, A.; Marin-Neto, J. A.; J. Chem. Res. 2010, $375,1388$.

4. Bermudez, J.; Davies, C.; Simonazzi, A.; Real, J. P.; Palma, S.; Acta Trop. 2016, 156, 1.

5. Wilkinson, S. R.; Kelly, J. M.; Expert Rev. Mol. Med. 2009, 11, 31.

6. Tapia, R. A.; Salas, C. O.; Vázquez, K.; Espinosa-Bustos, C.; Soto-Delgado, J.; Varela, J.; Birriel, E.; Cerecetto, H.; González, M.; Paulino, M.; Org. Synth. 2014, 24, 3919.

7. Menezes, J. C.; Vaz, L. B.; Vieira, P. M. A.; Fonseca, K. S.; Carneiro, C. M.; Taylor, J. G.; Molecules 2014, 20, 43.

8. Moreira, D. R.; Leite, A. C. L.; Cardoso, M. V.; Srivastava, R. M.; Hernandes, M. Z.; Rabello, M. M.; da Cruz, L. F.; Ferreira, R. S.; de Simone, C. A.; Meira, C. S.; Guimaraes, E. T.; Chem. Med. Chem. 2014, 9, 177.

9. Olmo, F.; Gómez-Contreras, F.; Navarro, P.; Marín, C.; Yunta, M. J.; Cano, C.; Campayo, L.; Martín-Oliva, D.; Rosales, M. J.; Sánchez-Moreno, M.; Eur. J. Med. Chem. 2015, 106, 106.

10. Gómez-Ayala, S.; Castrillón, J. A.; Palma, A.; Leal, S. M.; Escobar, P.; Bahsas, A.; Bioorg. Med. Chem. 2010, 18, 39. 
11. Caputto, M. E.; Ciccarelli, A.; Frank, F; Moglioni, A. G.; Moltrasio, G. Y.; Vega, D.; Lombardo, E.; Finkielsztein, L. M.; Eur. J. Med. Chem. 2012, 55, 63.

12. Cardoso, M. V. O.; de Siqueira, L. R.; da Silva, E. B.; Costa, L. B.; Hernandes, M. Z.; Rabello, M. M.; Ferreira, R. S.; da Cruz, L. F.; Moreira, D. R.; Pereira, V. R.; de Castro, M. C.; Eur. J. Med. Chem. 2014, 86, 48.

13. da Silva, R. B.; Loback, V. B.; Salomão, K.; de Castro, S. L.; Wardell, J. L.; Wardell, S. M.; Costa, T. E.; Penido, C.; de Henriques, M. D.; Carvalho, S. A.; da Silva, E. F.; Molecules 2013, $18,57$.

14. Soares, F. A.; Sesti-Costa, R.; da Silva, J. S.; de Souza, M. C.; Ferreira, V. F.; Santos, F. D.; Monteiro, P. A.; Leitao, A.; Montanari, C. A.; Bioorg. Med. Chem. 2013, 23, 4597.

15. Romanha, A. J.; Castro, S. L.; Soeiro, M. D.; Lannes-Vieira, J.; Ribeiro, I.; Talvani, A.; Bourdin, B.; Blum, B.; Olivieri, B.; Zani, C.; Spadafora, C.; Mem. Inst. Oswaldo Cruz 2010, 105, 233.

16. Elias, P. R.; Coelho, G. S.; Xavier, V. F.; Sales Junior, P. A.; Romanha, A. J.; Murta, S. M. F.; Carneiro, C. M.; Camilo, N. S.; Hilário, F. F.; Taylor, J. G.; Molecules 2016, 21, 1342.

17. Papadopoulou, M. V.; Bloomer, W. D.; Rosenzweig, H. S.; Wilkinson, S. R.; Kaiser, M.; Eur. J. Med. Chem. 2014, 87, 79.

18. Gomes, P. A. M.; Oliveira, A. R.; Cardoso, M. V. O.; Santiago, E. F.; Barbosa, M. O.; de Siqueira, L. R.; Moreira, D. R.; Bastos, T. M.; Brayner, F. A.; Soares, M. B.; Mendes, A. P. O.; Eur. J. Med. Chem. 2016, 111, 46.

19. Ishii, M.; Jorge, S. D.; de Oliveira, A. A.; Palace-Berl, F.; Sonehara, I. Y.; Pasqualoto, K. F.; Tavares, L. C.; Bioorg. Med. Chem. 2011, 19, 301.
20. dos Santos Filho, J. M.; Leite, A. C.; de Oliveira, B. G.; Moreira, D. R.; Lima, M. S.; Soares, M. B.; Leite, L. F.; Bioorg. Med. Chem. 2009, 17, 91.

21. de Andrade, P.; Galo, O. A.; Carvalho, M. R.; Lopes, C. D.; Carneiro, Z. A.; Sesti-Costa, R.; de Melo, E. B.; Silva, J. S.; Carvalho, I.; Bioorg. Med. Chem. 2015, 23, 26.

22. Chakroborty, S.; Bhanja, C.; Jena, S.; Heterocycl. Commun. 2013, 19, 79.

23. Li, Z.; Wen, G.; Fu, R.; Yang, J.; J. Chem. Res. 2016, 40, 643.

24. For a recent review see: Galenko, A. V.; Khlebnikov, A. F.; Novikov, M. S.; Pakalnis, V. V.; Rostovskii, N. V.; Russ. Chem. Rev. 2015, 84(4), 335.

25. Wheeler, T. S.; Org. Synth. 1952, 72.

26. Rao, P. S.; Kurumurthy, C.; Veeraswamy, B.; Poornachandra, Y.; Kumar, C. G.; Narsaiah, B.; Bioorg. Med. Chem. Lett. 2014, 24(5), 1349.

27. Molinspiration Cheminformatics, v2015.01, Bratislava University, Slovak Republic, 1986. Available at http://www. molinspiration.com/, accessed on September 20, 2016.

28. Livingston, M. J.; Chick, M. F.; Shealy, E. O.; Beam, C. F.; J. Heterocycl. Chem. 1982, 19, 215.

29. Patonay, T.; Boganr, R.; Tetrahedron 1984, 40, 2555.

30. Gothelf, K. V.; Torssell, K. B. G.; Acta Chem. Scand. 1994, 48, 61.

31. Shah, M.; Patel, P.; Korgaokar, S.; Parekh, H.; Indian J. Chem. 1996, 35, 1282.

Submitted: April 1, 2017

Published online: July 13, 2017 